Pacific Journal of Mathematic 


\title{
EXTENSIONS OF THE MAXIMAL IDEAL SPACE OF A FUNCTION ALGEBRA
}

\author{
J-E. BJöRK
}

\begin{abstract}
Let $A$ be a function algebra with its maximal ideal space $M_{A}$. Let $B$ be a function algebra such that $A \subset B \subset C\left(M_{A}\right)$. What can be said about $M_{B}$ ? We prove that $M_{A}=M_{B}$ if every point $x \in M_{A}$ has a fundamental neighborhood system $\{W\}$ such that the topological boundary $b W$ of each $W$ is contained in the Choquet boundary of $A$ or if $A$ is a normal function algebra. The first condition is satisfied if $M_{A}$ is a one dimensional topological space. Let $H(A)$ be the function algebra on $M_{A}$ generated by all functions which are locally approximable in $A$. We prove that $M_{H(A)}=M_{A}$ and then we try to generalize this result. If $f \in C\left(M_{A}\right)$ is such that $f$ is locally approx̌imable in $A$ at every point where $f$ is different from zero then $M_{A}$ is the maximal ideal space of the function algebra generated by $A$ and $f$. We also look at closed subsets $F$ of $M_{A}$ such that $M_{H(F)}=F$ where $H(F)$ is the function algebra generated by restricting to $F$ all functions that are defined and locally approximable in $A$ in some neighborhood of $F$. These sets are called natural sets. We prove that there exists a smallest natural set $B(F)$ containing a closed set $F$ in $M_{A}$ and that the Silov boundary of $H(B(F))$ is contained in $F$. We also find conditions that guarantee that a closed set in $M_{A}$ is a natural set.
\end{abstract}

If $X$ is a set and $f$ is a complex-valued function defined on $X$ then $|f|_{V}=\sup \{\mid f(x) \| x \in V\}$ for every $V \subset X$ and $f_{V}$ is the restriction of $f$ to $V$. If $V$ is a subset of a topological space $X$ then $b V$ is the topological boundary of $V$ in $X$. If $A$ is a function algebra we denote by $M_{A}$ its maximal ideal space, and $S_{A}$ its Shilov boundary. A point $x \in M_{A}$ is a strong boundary point in $A$ if $\{x\}=\cap P(f)$, where $P(f)$ are peak sets of $A$ in $M_{A}$. We shall use the wellknown fact that $S_{A}$ is the closure of the strong boundary points of $A$ in $M_{A}$. If $F$ is a closed set in $M_{A}$ then $\operatorname{Hull}_{A}(F)=\left\{\left.x \in M_{A}|| f(x)|\leqq| f\right|_{F}\right.$ for every $f \in A\}$. If $x \in \operatorname{Hull}_{A}(F)$ we say thet $F$ is a support of $x$. A minimal support of $x$ is a support $F$ of $x$ such that no proper closed subset of $F$ is a support of $x$. Now we have the principle of minimal supports. Let $F$ be a minimal support of $x$. Suppose $\left\{f_{n}\right\} \in A$ is such that $\left|f_{n}\right|_{F} \leqq K$ for some constant $K$ independent of $n$ and $\lim \left|f_{n}\right|_{W \cap F}=$ 0 , where $W$ is an open subset of $M_{A}$ such that $W \cap F$ is not empty. Then it follows that $\lim f_{n}(x)=0$. If $F$ is a closed set in $M_{A}$ then $A_{F}$ is the function algebra on $F$ generated by functions $f \in C(F)$ such that $f=g$ on $F$ for some $g \in A$. Now $M_{A_{F}}$ can be identified with 
Hull $_{A}(F)$. If $F$ is a closed set in $M_{A}$ such that $F=\operatorname{Hull}_{A}(F)$ we say that $F$ is an $A$-convex set. $A$ is a convex function algebra if every closed set in $M_{A}$ is $A$-convex. If $B$ is a function algebra on $M_{A}$ such that $A \subset B$ then the maximal ideal space $M_{B}$ contains $M_{A}$ and $S_{B} \subset M_{A}$. If $x \in M_{B}$ there exists a point $y(x) \in M_{A}$ such that $f(x)=f(y(x))$ for $f \in A$. If $V$ is a subset of $M_{A}$ we put $\{V\}_{B}=\left\{x \in M_{B} \mid y(x) \in V\right\}$. The set $\{V\}_{B}$ is called the fiber of $V$ in $M_{B}$. The correspondence between points $x$ in $M_{A}$ and the fibers $\{x\}_{B}$ is continuous in the following way: Let $W$ be an open neighborhood of $\{x\}_{B}$ in $M_{B}$ for some point $x \in M_{A}$. Then there exists a neighborhood $V$ of $x$ in $M_{A}$ such that $\{V\}_{B} \subset W$. If $W$ is an open set in $M_{A}$ then $H_{0}(W)=\{f \in C(W) \mid f$ is locally approximable in $A$ at every point in $W$, i.e., if $x \in W$ there exists a neighborhood $V \subset W$ of $x$ and $\left\{g_{n}\right\} \in A$ such that $\left.\lim \left|g_{n}-f\right|_{V}=0.\right\}$. We put $H_{0}(A)=H_{0}\left(M_{A}\right)$ and $H(A)$ is the function algebra generated by $H_{0}(A)$ on $M_{A}$. If $F$ is a closed set in $M_{A}$ then $H_{0}(F)=\{f \in C(F) \mid f=g$ on $F$ for some $g \in H_{0}(V)$, where $V$ is some neighborhood of $\left.F\right\}$. We let $H(F)$ be the function algebra on $F$ generated by $H_{0}(F)$. We shall now discuss the results of this paper. The general problem which interests us here is the following: Let $A$ be a function algebra with its maximal ideal space $M_{A}$. Let $B$ be a function algebra such that $A \subset B \subset C\left(M_{A}\right)$. What can be said about $M_{B}$ ? In Lemma 1 we give the well-known construction which shows that $M_{B}$ in general is strictly larger than $M_{A}$. A point $x \in M_{A}$ is a stationary point if $\{x\}_{B}=\{x\}$ for every $B$ such that $A \subset B \subset C\left(M_{A}\right) . \quad A$ is a resistent function algebra if $M_{A}$ consists of stationary points. In Theorem 2 we prove that $A$ is a resistent function algebra if every point $x \in M_{A}$ has a fundamental neighborhood system $\{W\}$ such that $\{b W\}$ consist of stationary points. We remark here that the Choquet boundary of $A$ is contained in the set of stationary points and that $A$ is resistent if $M_{A}=[0,1]$. A function algebra $A$ on a compact set $X$ is regular if $A$ separates points from closed subsets of $X$. It is wellknown that if $X=M_{A}$ then $A$ is normal, i.e., $A$ separates disjoint closed sets. In Theorem 4 we prove that if $A$ is a regular function algebra on $X$ then $X$ consists of stationary points when we consider $X$ as a closed subset of $M_{A}$. We remark that if $A$ is a normal function algebra on $X$ then $X=M_{A}$. The rest of this paper is mostly devoted to a study of relations between $A$ and $H(A)$. We have never introduced the general concept of $A$-holomorphic functions as is done in [3]. We wish to point out that our methods come almost entirely from [3] and [4]. Our proof of Theorem 5 uses an argument which is essentially the same as in Lemma 3.1, p. 368, in [3]. We point out that Theorem 7 gives a proof of Rado's Theorem: Let $f \in C(F)$ where $F$ is a polynomially convex compact set in the complex plane. Assume that $f$ is 
analytic if $f$ is different from zero. Then it follows that $f$ is analytic in the interior of $F$ and hence $f \in P(F)$, i.e., $f$ can be uniformly approximated by polynomials on $F$. In Theorem 8 we prove that if $H(A)$ is a resistent function algebra then $A$ is a resistent function algebra. We also discuss the general problem of determining 'domains of holomorphy' in general function algebras. A closed set $F$ in $M_{A}$ is a natural set if $M_{I I(F)}=F$. The main result about natural sets is contained in Theorem 10 which was essentially wellknown in [3]. Every closed subset. $F$ of $M_{A}$ is contained in a smallest natural set $B(F)$, the barrier of $F$. We have also introduced the set $\hat{F}=$ $\left\{y \in M_{A} \mid\{y\}_{H(F)} \cap M_{H(F)}\right.$ is not empty\}. We know that $\hat{F} \subset B(F)$ and in general the inclusion is strict. ${ }^{1}$ Theorem 12 is essentially wellknown in [5] but we believe our proof is different.

1. Definition 1. A function algebra $A$ is resistent if $M_{B}=M_{A}$ for every function algebra $B$ such that $A \subset B \subset C\left(M_{A}\right)$.

Lemma 1. A resistent function algebra is convex.

Proof. Let $A$ be a function algebra such that $\operatorname{Hull}_{A}(F)-F$ is not empty for some closed set $F$ in $M_{A}$. Let $B=\left\{g \in C\left(M_{A}\right) \mid g_{F} \in A_{F}\right\}$. Obviously $A \subset B \subset C\left(M_{A}\right)$ and now we prove that $M_{A} \neq M_{B}$. Let $x \in \operatorname{Hull}_{A}(F)-F$. If $g \in B$ we can find $\left\{f_{n}\right\} \in A$ such that $\lim \left|g-f_{n}\right|_{F}=$ 0 . Now we put $\hat{x}(g)=\lim f_{n}(x)$. It is easily seen that $\hat{x}$ is a well defined complex-valued homomorphism on $B$. Hence there exists a point $y \in M_{B}$ such that $\hat{x}(g)=g(y)$ for $g \in B$. In particular $f(x)=$ $\hat{x}(f)=f(y)$ for $f \in A$. If $M_{A}=M_{B}$ it follows that $\hat{x}(g)=g(x)$ for $g \in B$. But now we choose $g \in B$ such that $g(x)=1$ while $g=0$ on $F$ and obtain a contradiction. Hence $M_{A} \neq M_{B}$ and the lemma follows.

Lemma 2. Let $A$ be a convex function algebra and let

$$
A \subset B \subset C\left(M_{A}\right) \text {. }
$$

Then the fibers $\{x\}_{B}$ are connected in $M_{B}$ for every point $x \in M_{A}$.

Proof. Suppose that some fiber $(x)_{B}$ is disconnected in $M_{B}$. Hence there exists a closed component $G$ of $\{x\}_{B}$ such that $G \subset M_{B}-M_{A}$. Now we can find a closed neighborhood $W$ of $G$ in $M_{B}$ such that $b W \cap\{x\}_{B}$ is empty and $W \subset M_{B}-M_{A}$. Let $F=\left\{y \in M_{A} \mid\{y\}_{B} \cap b W\right.$ is not empty\}. Obviously $F$ is a closed subset of $M_{A}$ such that $x \notin F$. Let $y \in G$, then the local maximum principle shows that $|g(y)| \leqq|g|_{b W}$ for $g \in B$. It follows that $|f(x)| \leqq|f|_{F}$ for $f \in A$, hence $x \in \operatorname{Hull}_{A}(F)$,

${ }^{1} \mathrm{I}$ am indebted to the referee for giving an example where $F \neq B(\hat{F})$. 
a contradiction to the fact that $A$ is a convex function algebra.

Theorem 1. Let $V$ be a closed A-convex subset of $M_{A}$ such that $A_{V}$ is resistent. Let $f \in C\left(M_{A}\right)$ be such that $f=0$ in $M_{A}-V$, then $M_{A(f)}=M_{A}$.

Proof. Assume that $D=M_{A(f)}-M_{A}$ is not empty. Let $x \in D$ and choose a minimal support $F$ of $x$ such that $F \subset M_{A}$. Now $F \subset V$ is impossible since $A_{V}$ is a resistent function algebra. Because $f=0$ in $M_{A}-V$ the principle of minimal supports shows that $f(x)=0$. Choose $y \in M_{A}$ such that $g(x)=g(y)$ for $g \in A$. Since $y$ and $x$ are different points of $M_{A(f)}$ it follows that $f(y)$ must be different from zero, hence $y \in V$. We have now proved that $D \subset\{V\}_{A(f)}$. Now Lemma 1 shows that $A_{V}$ is a convex function algebra and Lemma 2 can be applied to show that $\{z\}_{A(f)}$ are connected in $M_{A(f)}$ for every $z \in V$. In particular $\{y\}_{A(f)}$ has no isolated points in $M_{A(f)}$. Since $D$ is an open subset of $M_{A(f)}$ we can find $x_{1} \in D \cap\{y\}_{A(f)}$ such that $x_{1} \neq x$. But now we get $f\left(x_{1}\right)=f(x)=0$ and then $x$ and $x_{1}$ are not different points in $M_{A(f)}$, a contradiction.

Definition 2. A point $x \in M_{A}$ is stationary if $\{x\}_{B}=\{x\}$ for every function algebra $B$ such that $A \subset B \subset C\left(M_{A}\right)$.

THeOREM 2. Let $A$ be a function algebra such that every point $x \in M_{A}$ has a fundamental neighborhood system $\{W\}$ such that each $b W$ consists of stationary points, then $A$ is a resistent function algebra.

Proof. Suppose that $B$ is a function algebra such that

$$
A \subset B \subset C\left(M_{A}\right)
$$

and assume that $D=M_{B}-M_{A}$ is not empty. Let $z \in D$ and choose $y \in M_{A}$ such that $f(z)=f(y)$ for $f \in A$. Choose an open neighborhood $V$ of $y$ in $M_{A}$ such that $b V$ consists of stationary points. Let $W$ be a closed $B$-convex neighborhood of $z$ in $M_{B}$ such that $W \subset D$. Now $\{V\}_{B} \cap W$ is open and closed in $W$. We apply Shilov's Idempotent Theorem to the function algebra $B_{W}$. Hence we find $\left\{f_{n}\right\} \in B$ such that $\lim \left|f_{n}-1\right|_{W \cap(V\}_{B}}=0$ while $\lim \left|f_{n}\right|_{W-\{V\}_{B}}=0$. Choose a minimal support $F$ of $z$ such that $F \subset b W$. It follows from the principle of minimal supports that $F \subset b W \cap\{V\}_{B}$. Now we let $V$ shrink to $y$ in $M_{A}$ and it follows that $z \in \operatorname{Hull}_{B}\left(b W \cap\{y\}_{B}\right)$. This holds for every $z \in D \cap\{y\}_{B}$ when $W$ is a closed $B$-convex neighborhood of $z$ such that $W \subset D$. Now we choose a strong boundary point $x \in D \cap\{y\}_{B}$ of the function algebra $B_{\{y\}_{B}}$ to obtain a contradiction. 
Definition 4. A point $x \in M_{A}$ is locally regular if there exists a neighborhood $V$ of $x$ such that to every $y \in V-\{x\}$ there exists $f \in A$ with $f=0$ in a neighborhood of $y$ and $f(x)=1$.

Theorem 3. A locally regular point is a stationary point.

Proof. Let $x \in M_{A}$ be a locally regular point. Let $B$ be a function algebra such that $A \subset B \subset C\left(M_{A}\right)$. Let $D=M_{B}-M_{A}$ and assume that $\{x\}_{B} \cap D$ is not empty. Let $V$ be an open neighborhood of $x$ in $M_{A}$ such that to every $y \in V-\{x\}$ there exists $f \in A$ with $f=0$ in a neighborhood of $y$ and $f(x)=1$. Let $z \in\{x\}_{B} \cap D$ and choose a closed neighborhood $W$ of $z$ in $M_{B}$ such that $W \subset D \cap\{V\}_{B}$. Let $F$ be a minimal support of $z$ such that $F \subset b W$. It follows now that $F \subset\{x\}_{B}$ holds. Hence $z \in \operatorname{Hull}_{B}\left(b W \cap\{x\}_{B}\right)$ and we obtain a contradiction if we choose a suitable point $z \in D \cap\{x\}_{B}$. Hence $\{x\}_{B} \cap D$ must be empty and it follows that $x$ is a stationary point.

THEOREM 4. Let $A$ be a regular function algebra on a compact set $X$. Then every point $x \in X \cap M_{A}$ is a stationary point.

Proof. Let $x \in X \cap M_{A}$ and put $R(x)=\left\{y \in M_{A} \mid\right.$ there exists $g \in A$ with $g=0$ in a neighborhood of $y$ and $g(x)=1\}$. We shall now prove that $R(x)=M_{A}-\{x\}$ and then it follows from Theorem 3 that $x$ is a stationary point. Let $y \in M_{A}-\{x\}$ and choose $g \in A$ such that $g(y)=1$ and $g(x)=0$. Let $V=\left\{z \in M_{A}|| g(z) \mid>1 / 2\right\}$ and let $W=\{z \in X \| g(z) \leqq 1 / 2\}$. We choose $f \in A$ such that $f=0$ on $X-W$ and $f(x)=1$. If $z \in V$ we can choose a minimal support $F$ of $z$ such that $F \subset X$. Obviously $F \cap(X-W)$ is not empty and the principle of minimal supports implies that $f(z)=0$. Hence $f=0$ on $V$ and $f(x)=1$, i.e., $y \in R(x)$.

Theorem 5. Let $F$ be a closed subset of $M_{A}$ and let $f \in C M_{A}$ be such that $f$ is locally approximable in $A$ at every point in $M_{A}-F$. Then $M_{A(f)}-M_{A} \subset\left\{\operatorname{Hull}_{A}(F)\right\}_{A(f)}$.

Proof. Let $D=M_{A(f)}-M_{A}$. Let $K=\operatorname{Hull}_{A(f)}(b D)$ and let $C=$ $A(f)_{K}$. We have $D \subset K=M_{C}$ and $b D$ contains the Shilov boundary of $C$. Let $x \in b D$ be a strong boundary point of $C$. Assume that $x \in M_{A}-F$. Choose a closed neighborhood $V$ of $x$ in $M_{A}$ such that there exists $\left\{g_{n}\right\} \in A$ with $\lim \left|g_{n}-f\right|_{V}=0$. Now we choose $h \in C$ such that $h(x)=|h|_{K}=1$ and $\{x \in K \| h(x) \mid \geqq 1 / 2\} \subset\{V\}_{A(f)}$. Let

$$
D_{1}=\{x \in D \| h(x) \mid>1 / 2\} \text {. }
$$

The topological boundary $b D_{1}$ of $D_{1}$ in $K$ is obviously contained in the set $T=\{x \in b D \| h(x) \mid \geqq 1 / 2\} \cup\{x \in K \| h(x) \mid=1 / 2\}$. Choose a point 
$x_{1} \in D_{1}$. Now the local maximum principle shows that we can find a minimal support $F$ of $x_{1}$ in $C$ such that $F \subset T$. Since $\left|h\left(x_{1}\right)\right|>1 / 2$ it follows that $F \cap b D$ contains an open subset of $F$. Since $F \subset T \subset\{V\}_{A(f)}$ we have $|g|_{F} \leqq|g|_{V}$ for $g \in A$. Now $\lim \left|g_{n}-f\right|_{F \cap b D} \leqq \lim \left|g_{n}-f\right|_{V}=$ 0 and the principle of minimal supports shows that $\lim g_{n}\left(x_{1}\right)=f\left(x_{1}\right)$ holds. Now we also have $x_{1} \in\left\{y_{1}\right\}_{A(f)}$ for some point $y_{1} \in V$. Hence $f\left(y_{1}\right)=\lim g_{n}\left(y_{1}\right)=g_{n}\left(x_{1}\right)=f\left(x_{1}\right)$ and then $x_{1}$ and $y_{1}$ cannot be different points in $M_{A(f)}$, a contradiction. We have now proved that every strong boundary point of $C$ must belong to $F$. It follows that $S_{C} \subset F$ and hence $M_{A(f)}-M_{A} \subset \operatorname{Hull}_{A(f)}(F)$. This implies that $M_{A(f)}-M_{A} \subset\left\{\operatorname{Hull}_{A}(F)_{A(f)}\right.$ •

Lemma 3. Let $A$ be a function algebra on a compact set $X$. Let $F$ be a closed subset of $X$. Then there exists a point $x \in F$ such that if $m$ is a representing measure of $x$ in $A$ with $m(F)=1$ then $m=e_{x}$, i.e., $m$ is the unit point mass at $x$.

Proof. Choose a strong boundary point $x \in F$ of the function algebra $A_{F}$.

Theorem 6. Let $A \subset B \subset C\left(M_{A}\right)$. Let $f \in B$ be such that $f \in H_{0}(A)$. Then $f$ is constant on each fiber $\{x\}_{B}$ for $x \in M_{A}$.

Proof. If $x \in M_{B}$ we denote by $y(x)$ the point in $M_{A}$ such that $x \in\{y(x)\}_{B}$. Let $d(x)=|f(x)-f(y(x))|$ and assume that $d(x)$ is different from zero. Let $F=\left\{x \in M_{B} \mid d(z)=\|d\|=\sup d(z)\right\}$. Obviously $F$ is a closed subset of $M_{B}$ and $F \cap M_{A}$ is empty. Let $x \in F$ and choose an open neighborhood $V$ of $y(x)$ in $M_{A}$ such that there exists $\left\{g_{n}\right\} \in A$ with $\lim \left|g_{n}-f\right|_{V}=0$. Choose now a closed neighborhood $W$ of $x$ in $M_{B}$ such that $W \subset\{V\}_{B} \cap\left(M_{B}-M_{A}\right)$. Let $T$ be a minimal support of $x$ such that $T \subset b W$. Now we can find a positive measure on $T$ such that $g(x)=\int g d m$ from $g \in B$. It follows that $\left|f(x)-g_{n}(y(x))\right|=$ $\left|f(x)-g_{n}(x)\right| \leqq \int\left|f-g_{n}\right| d m$ for every $n$. Hence we also get

$$
|f(x)-f(y(x))| \leqq \int|f(z)-f(y(z))| d m(z) .
$$

It follows that $|f(z)-f(y(z))|=\|d\|$ for every $z \in T$, hence $T \subset F$. We have now proved that $x \in \operatorname{Hull}_{B}(b W \cap F)$ for every $x \in F$ and every closed neighborhood $W$ of $x$ such that $W \subset\left(M_{B}-M_{A}\right)$. Now we derive a contradiction from Lemma 3 .

Theorem 7. Let $f \in C\left(M_{A}\right)$ and suppose that $f$ is locally approximable in $A$ at every point where $f$ is different zero. Then $M_{A(f)}=M_{A}$ and Hull $_{A}(F)=$ Hull $_{A(f)}(F)$ for every closed subset $F$ of $M_{A}$. 
Proof. Let $F$ be a closed subset of $M_{A}$ such that $F=\operatorname{Hull}_{A(f)}(F)$. Let us put $G=\operatorname{Hull}_{A}(F)$ and assume that $D=G-F$ is not empty. Let $C=A(f)_{G}$. We see that the Shilov boundary $S_{C}$ of $C$ meets $D$. Hence we can find $x \in D$ such that $x$ is a strong boundary point of $C$. Let us assume that $f(x) \neq 0$. Choose a closed neighborhood $V \subset\left(M_{1}-F\right)$ of $x$ in $M_{A}$ such that there exist $\left\{g_{n}\right\} \in A$ with lim $\left|g_{n}-f\right|_{V}=0$. Now we choose $h \in C$ such that if $P(h)=\{x \in G \mid h(x)=$ $\left.|h|_{G}\right\}$ then $x \in P(h)$ and $P(h) \subset V$ with $P(h) \cap b V$ empty. Since $h \in C$ we can find $\left\{h_{n}\right\} \in A$ with $\lim \left|h_{n}-h\right|_{V \cap G}=0$. Now the local maximum principle shows that $|g(x)| \leqq|g|_{b V \cap G}$ for $g \in A$. It follows that $\mid h(x)=$ $\lim \left|h_{n}(x)\right| \leqq \lim \left|h_{n}\right|_{b V \cap G}=|h|_{b V \cap G}$, contradiction to the fact that $P(h) \cap b V$ is empty. Hence we have proved that if $x \in D$ is a strong boundary point of $C$ then $f(x)=0$. If $x \in D$ we can choose a minimal support $T$ of $x$ such that $T \subset S_{C}$. Since $F=\operatorname{Hull}_{A(f)}(F)$ it follows that $T \cap D$ is not empty. Since $f=0$ on $S_{C} \cap D$ it follows from the principle of minimal supports that $f(x)=0$. Hence we have proved that $f=0$ on $D$. But then $A(f)_{D}=A_{D}$ and it follows easily that $D$ cannot contain any strong boundary point of $C$. Hence $S_{C} \subset F$ which shows that $D$ must be empty. We have now proved that Hull ${ }_{A}(F)=$ Hull $_{A(f)}(F)$ for every closed subset $F$ of $M_{A}$. In particular we see that $Z(f)=\left\{x \in M_{A} \mid f(x)=0\right\}$ is an $A$-convex set and using Theorem 5 it follows easily that $M_{A}=M_{A(f)}$.

CoRollary 1. $M_{A}=M_{H(A)}$ and $\operatorname{Hull}_{A}(F)=\operatorname{Hull}_{H(A)}(F)$ for every closed subset $F$ of $M_{A}$.

THEOREM 8. If $H(A)$ is a resistent function algebra then $A$ is a resistent function algebra.

Proof. If $A$ is not a resistent function algebra we can find $g_{1} \cdots g_{k} \in C\left(M_{A}\right)$ such that $g_{1} \cdots g_{k}$ have no common zero on $M_{A}$ while $g_{i}(z)=\cdots=g_{k}(z)=0$ for some point $z \in M_{A\left(g_{1} \cdots g_{k}\right)}$. Because $H(A)$ is resistent we can find $h_{1} \cdots h_{k}$, where each $h_{i}$ is a polynomial in $g_{1} \cdots g_{k}$ with coefficients in $H_{0}(A)$, such that $\left|h_{1} g_{1}+\cdots+h_{k} g_{k}-1\right|_{M_{A}}<1 / 2$. Let $h_{i}=\sum f_{i v} g^{v}$, where $v$ runs over a finite set of multi-indices $\left(v_{1} \cdots v_{k}\right)$ and $g^{v}=g_{1}^{v_{1}} \cdots g_{k}^{v}$. Each $f_{i v} \in H_{0}(A)$ and we define $f_{i v}$ on $M_{A\left(g_{1} \cdots g_{k}\right)}$ by letting $f_{i v}$ be constant on each fiber of $M_{A\left(g_{1} \cdots g_{k}\right)}$ over points of $M_{A}$. Each $g^{v}$ is defined on $M_{A\left(g_{1} \cdots g_{k}\right)}$ in the usual way. In this way we can extend each $h_{i}$ to $M_{A\left(g_{1} \cdots g_{k}\right)}$. Call these extensions $H_{1} \cdots H_{k}$. It is easily seen that $H=H_{1} g_{1}+\cdots+H_{k} g_{k}$ is locally approximable in $A\left(g_{1} \cdots g_{k}\right)$ on $M_{A\left(g_{1} \cdots g_{k}\right)}$. Now $H(z)=0$ while $|H-1|_{M_{A}}<1 / 2$ and since $M_{4}$ contains the Shilov boundary of $A\left(g_{1} \cdots g_{k}\right)$ we derive a contradiction from Corollary 1.

THEOREM 9. Let $f \in C\left(M_{A}\right)$ be such that $f^{n}+a_{1} f^{n-1}+\cdots+a_{n}=0$ 
on $M_{A}$ where $a_{1} \cdots a_{n} \in A$, then $M_{A}=M_{A(f)}$.

Proof. Let $g=n f^{n-1}+(n-1) a_{1} f^{n-2}+\cdots+a_{n-1}$. It is well known that $f$ is locally approximable in $A$ at every point $x \in M_{A}$ where $g(x)$ is different from zero. (See [1], Th. 3.2.5, p. 71.) It follows that $g$ is locally approximable in $A$ at every point where $g$ is different from zero. Now Theorem 7 shows that $Z(g)$ is $A$-convex and then Theorem 5 shows that $M_{A(f)}-M_{A} \subset\{Z(g)\}_{A(f)}$. Let us put $B=A_{Z(g)}$, then $M_{B}=Z(g)$ and the restriction of $f$ to $M_{B}$ satisfies the equation $n f^{n-1}+(n-1) b_{1} f^{n-2}+\cdots+b_{n-1}=0$ where $b_{i} \in B$ are the restrictions of $a_{i}$ to $Z(g)$. Since $M_{A(f)}-M_{A} \subset\{Z(g)\}_{A(f)}$ we see that $M_{B(f)}-M_{B}$ is not empty if $M_{A(f)}-M_{A}$ is not empty. Hence we can use induction over $n$ to prove that $M_{A(f)}=M_{A}$.

Let $A$ be a function algebra. If $F$ is a closed subset of $M_{A}$ we have defined the function algebra $H(F)$. We are now interested in the maximal ideal space of $H(F)$.

Definition. If $F$ is a closed subset of $M_{A}$ we put $\hat{F}=$ $\left\{y \in M_{A} \mid\{y\}_{H(F)} \cap M_{H(F)}\right.$ is not empty $\}$.

Definition. A natural set in $M_{A}$ is a closed subset $F$ of $M_{A}$ such that $F=M_{H(F)}$.

Lemma 4. $\left(\cap F_{u}\right)^{\wedge} \subset \cap \hat{F}_{a}$ for every family $\left\{F_{a}\right\}$ of closed subsets of $M_{A}$.

Proof. Let $y \in M_{A}$ be such that $y \in\left(\cap F_{a}\right)^{\wedge}$. Hence there exists a complex-valued homomorphism $C$ of $H\left(\cap F_{a}\right)$ such that $C(g)=g(y)$ for $g \in A$. If $f \in H\left(F_{a}\right)$ the restriction of $f$ to $\cap F_{a}$ obviously gives an element of $H\left(\cap F_{a}\right)$. Hence $C$ can be restricted to $H\left(F_{a}\right)$ and we obtain a complex-valued homomorphism of $H\left(F_{a}\right)$ such that $C(g)=g(y)$ for $g \in A$.

Theorem 10. Let $F$ be a closed subset of $M_{A}$ such that $F=\hat{F}$, then $M_{H(F)}=F$.

Proof. Let $f \in H_{0}(F)$ and define $d(x)=|f(x)-f(y(x))|$ on $M_{H(F)}$ where $y(x)$ is the point in $F$ such that $g(x)=g(y(x))$ for $g \in A$. Assume that $d$ is not identical zero. Let $D=\left\{x \in M_{H(F)} \mid d(x)>0\right\}$. Obviously $D \cap F$ is empty and hence $D$ lies off the Shilov boundary of $H(F)$. Hence $D \subset K=\operatorname{Hull}_{I I(F)}(b D)$. Let us put $C=H(F)_{K}$ and choose $x \in b D$ such that $x$ is a strong boundary point of $C$. Choose a closed neighborhood $V$ of $y(x)$ in $M_{A}$ such that there exists $\left\{g_{n}\right\} \in A$ with $\lim \left|g_{n}-f\right|_{V \cap F}=0$. Now we choose $h \in C$ such that $h(x)=$ $|h|_{K}=1$ and $\{x \in K|| h(x) \mid \geqq 1 / 2\} \subset\{V \cap F\}_{H(F)}$. Now we obtain a con- 
tradiction using the same argument as in the final part of Theorem 5. Hence we have proved that if $f \in H_{0}(F)$ then $f$ is constant on each fiber $\{x\}_{H(F)}$ when $x \in F$. Since $H_{0}(F)$ is a dense subalgebra of $H(F)$ it follows that $F=M_{H(F)}$.

Corollary 2. If $\left\{F_{a}\right\}$ is a family of natural set of $M_{A}$ then $\cap F_{a}$ is a natural set.

Proof. Lemma 4 shows that $\left(\cap F_{a}\right)^{\wedge} \subset \cap \hat{F}_{a}=\cap F_{a}$ and then Theorem 10 implies that $\cap F_{a}$ is a natural set.

Definition. If $F$ is a closed subset of $M_{A}$ then $B(F)$ is the intersection of all natural sets containing $F . B(F)$ is called the barrier of $F$.

Corollary 2 shows that $B(F)$ is the smallest natural set containing a closed subset $F$ of $M_{A}$.

Lemma 5. Let $F$ be a natural set. Let $f \in H(F)$ and let $F_{1}=$ $\{x \in F|| f(x) \mid \leqq 1\}$. Then $F_{1}$ is a natural set.

Proof. Let $z \in M_{I I\left(F_{1}\right)}$. If $g \in H(F)$ the restriction of $g$ to $F_{1}$ gives an element of $H\left(F_{1}\right)$. It follows that $g(z)=g(y)$ for some point $y \in M_{H(F)}$ when $g \in H(F)$. In particular $f(z)=f(y)$ and since $|f(z)| \leqq|f| F_{1}$ it follows that $y \in F_{1}$. Hence we have proved that $F_{1}=\hat{F}_{1}$ and now Theorem 10 implies that $F_{1}$ is a natural set.

THEOREM 11. Let $F$ be a closed subset of $M_{A}$. Let $S(F)$ be the Shilov boundary of $H(B(F))$. Then $S(F) \subset F$.

Proof. Assume that $S\left(F^{\prime}\right)$ meets $B(F)-F$. Hence we can find $x \in B(F)-F$ such that $x$ is a strong boundary point of $H(B(F))$. Now we can choose $f \in H(B(F))$ such that $F_{1}=\{x \in B(F)|| f(x) \mid \leqq 1\}$ contains $F$ and omits the point $x$.

Lemma 5 shows that $F_{1}$ is a natural set, a contradiction to the fact that $B(F)$ is the smallest natural set containing $F$.

We finally give some examples of natural subsets of $M_{A}$.

Definition. An $A$-analytic polyhedron $P$ is a closed set in $M_{A}$ of the form $P=\left\{x \in V|| f_{a}(x) \mid \leqq 1\right.$ where $V$ is an open neighborhood of $P$ and $\left\{f_{a}\right\}$ is a family in $\left.H_{0}(V)\right\}$.

Theorem 12. An A-analytic polyhedron is a natural set. 
Proof. Let $U$ be an open neighborhood of $P$ and $W$ a closed set containing $U$ such that $W \subset V$. Now we can find finitely many $\left\{f_{a}\right\}$, say $f_{1} \cdots f_{k}$ such that $P_{1}=\left\{x \in W|| f_{i}(x) \mid \leqq 1, i=1 \cdots k\right\}$ is contained in $U$. Now we can prove that $P_{1}$ is a natural set using the same argument as in the final part of Theorem 5. Finally we let $U$ shrink to $P$ and obtain natural sets $\left\{P_{U}\right\}$ such that $P=\cap P_{U}$. Now Corollary 2 shows that $P$ is a natural set.

Definition. If $F$ is a closed subset of $M_{A}$ we put $R_{0}(F)=$ $\{h \in C(F) \mid h=f / g$ where $f, g \in A$ and $g$ has no zero on $F\}$.

We let $R(F)$ be the function algebra on $F$ generated by $R_{0}(F)$.

Definition. If $F$ is a closed subset of $M_{A}$ we put $\operatorname{Hull}_{R}(F)=$ $\left\{x \in M_{A} \mid g(x) \in g(F)\right.$ for $\left.g \in A\right\}$.

THEOREM 13. $M_{R(F)}=$ Hull $_{R}(F)$ for every closed set $F$ in $M_{A}$ and if $M_{R(F)}=F$ then $F$ is a natural set.

Proof. If $y \in M_{R(F)}$ we choose $x \in M_{A}$ such that $g(y)=g(x)$ for $g \in A$. It is easily seen that $x \in \operatorname{Hull}_{R}(F)$ and that $(f / g)(y)=f(x) / g(x)$ when $f / g \in R_{0}(F)$. Since $R_{0}(F)$ is dense in $R(F)$ it follows that $y$ is uniquely determined by $x$. Conversely if we choose $x \in$ Hull $_{R}(F)$ then the mapping $X ; f / g \rightarrow f(x) / g(x)$ is well defined on $R_{0}(F)$. We have $|f(x) / g(x)| \leqq\left.|f| g\right|_{F}$ for if $f(x)=g(x)$ while $|f / g|_{F}<1$ we see that $(g-f)$ is different from zero on $F$ and hence $(g-f)(x) \in(g-f)(F)$ is different from zero, a contradiction. Hence we can extend $X$ to $R(F)$ and we obtain a complex-valued homomorphism on $R(F)$ such that $g$ is mapped into $g(x)$ when $g \in A$. This proves that $M_{R(F)}=$ Hull $_{R}(F)$. If $M_{R(F)}=F$ then Corollary 1 can be applied to prove that $F$ is a natural set.

Acknowledgement. I wish to express here my deep gratitude to Professor C. E. Rickart whose original research on this subject has been the source for this paper.

\section{BIBLIOGRAPHY}

1. L. Hörmander, An introduction to complex analysis in several variables, D. Van Nostrand, New Jersey, 1966.

2. H. Rossi and R. Gunning, Analytic functions of several complex variables, PrenticeHall, 1965.

3. C. E. Rickart, Analytic phenomena in general function algebras, Pacific J. Math. 18 (1966), 361-377.

4. - The maximal ideal space of functions locally approximable in a function algebra, Proc. Amer. Math. Soc. 17 (1966), 1320-1326.

5. - Holomorphic convexity for general function algebras, Yale University, 1967.

Received June 30, 1967, and in revised form December, 18, 1967.

UNIVERSITY OF STOCKHOLM 


\section{PACIFIC JOURNAL OF MATHEMATICS}

\section{EDITORS}

\section{H. ROYDEN}

Stanford University

Stanford, California

\section{J. Dugundu}

Department of Mathematics University of Southern California Los Angeles, California 90007

RICHARD ARENS

University of California Los Angeles, California 90024

ASSOCIATE EDITORS
E. F. Beckenbach
B. H. NEUMANN
F. WoLF
K. YosidA

\section{SUPPORTING INSTITUTIONS}

\author{
UNIVERSITY OF BRITISH COLUMBIA \\ CALIFORNIA INSTITUTE OF TECHNOLOGY \\ UNIVERSITY OF CALIFORNIA \\ MONTANA STATE UNIVERSITY \\ UNIVERSITY OF NEVADA \\ NEW MEXICO STATE UNIVERSITY \\ OREGON STATE UNIVERSITY \\ UNIVERSITY OF OREGON \\ OSAKA UNIVERSITY \\ UNIVERSITY OF SOUTHERN CALIFORNIA
}

STANFORD UNIVERSITY

UNIVERSITY OF TOKYO

UNIVERSITY OF UTAH

WASHINGTON STATE UNIVERSITY

UNIVERSITY OF WASHINGTON

AMERICAN MATHEMATICAL SOCIETY
CHEVRON RESEARCH CORPORATION
TRW SYSTEMS

AMERICAN MATHEMATICAL SOCIETY

TRW SYSTEMS

NAVAL WEAPONS CENTER

Mathematical papers intended for publication in the Pacific Journal of Mathematics should be in typed form or offset-reproduced, double spaced with large margins. Underline Greek letters in red, German in green, and script in blue. The first paragraph or two must be capable of being used separately as a synopsis of the entire paper. It should not contain references to the bibliography. Manuscripts, in duplicate if possible, may be sent to any one of the four editors. All other communications to the editors should be addressed to the managing editor, Richard Arens, University of California, Los Angeles, California 90024.

Each author of each article receives 50 reprints free of charge; additional copies may be obtained at cost in multiples of 50 .

The Pacific Journal of Mathematics is published monthly. Effective with Volume 16 the price per volume (3 numbers) is $\$ 8.00$; single issues, $\$ 3.00$. Special price for current issues to individual faculty members of supporting institutions and to individual members of the American Mathematical Society: $\$ 4.00$ per volume; single issues $\$ 1.50$. Back numbers are available.

Subscriptions, orders for back numbers, and changes of address should be sent to Pacific Journal of Mathematics, 103 Highland Boulevard, Berkeley 8, California.

Printed at Kokusai Bunken Insatsusha (International Academic Printing Co., Ltd.), 7-17, Fujimi 2-chome, Chiyoda-ku, Tokyo, Japan.

PUBLISHED BY PACIFIC JOURNAL OF MATHEMATICS, A NON-PROFIT CORPORATION

The Supporting Institutions listed above contribute to the cost of publication of this Journal, but they are not owners of publishers and have no responsibility for its content or policies. 


\section{Pacific Journal of Mathematics \\ Vol. 27, No. 3}

March, 1968

Charles A. Akemann, Invariant subspaces of $C(G) \ldots \ldots \ldots \ldots \ldots \ldots . \ldots 41$

Dan Amir and Zvi Ziegler, Generalized convexity cones and their duals ... . 425

Raymond Balbes, On ( $J, M, \mathrm{~m})$-extensions of order sums of distributive

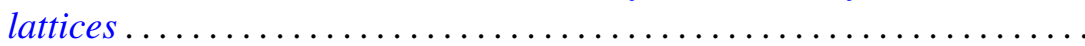

Jan-Erik Björk, Extensions of the maximal ideal space of a function algebra ........................................ 453

Frank Castagna, Sums of automorphisms of a primary abelian group ...... 463

Theodore Seio Chihara, On determinate Hamburger moment problems ..... .

Zeev Ditzian, Convolution transforms whose inversion function has complex

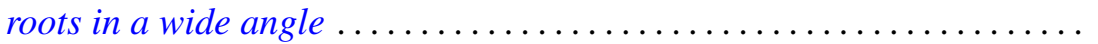

Myron Goldstein, On a paper of Rao .

Velmer B. Headley and Charles Andrew Swanson, Oscillation criteria for

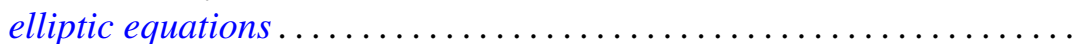

John Willard Heidel, Qualitative behavior of solutions of a third order nonlinear differential equation............................

Alan Carleton Hindmarsh, Pick's conditions and analyticity.............

Bruce Ansgar Jensen and Donald Wright Miller, Commutative semigroups

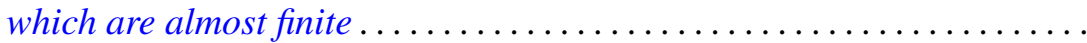

Lynn Clifford Kurtz and Don Harrell Tucker, An extended form of the

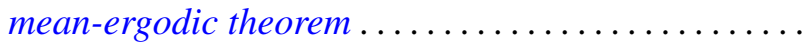

S. P. Lloyd, Feller boundary induced by a transition operator ...

Henry B. Mann, Josephine Mitchell and Lowell Schoenfeld, A new proof of the maximum principle for doubly-harmonic functions ...

Robert Einsohn Mosher, The product formula for the third obstruction ..

Sam Bernard Nadler, Jr., Sequences of contractions and fixed points ...

Eric Albert Nordgren, Invariant subspaces of a direct sum of weighted shifts...

Fred Richman, Thin abelian p-groups ...

Jordan Tobias Rosenbaum, Simultaneous interpolation in $\mathrm{H}_{2}$. II ...

Charles Thomas Scarborough, Minimal Urysohn spaces .

Malcolm Jay Sherman, Disjoint invariant subspaces..... .

Joel John Westman, Harmonic analysis on groupoids....

621

William Jennings Wickless, Quasi-isomorphism and TFM

Minoru Hasegawa, Correction to "On the convergence of resolvents of operators" 